Partnerschaft knapp 200 bis 300 deutsche Kommunisten aus den sowjetischen Gefängnissen entlassen wurden, um sie daraufhin mittels des Innenministeriums der UdSSR (NKWD) 1940 auf der Brücke von Brest-Litowsk den Nazis auszuliefern. Die politischen und ideologischen zeitgenössischen Gegner jedweden Totalitarismus im Allgemeinen und der kommunistischen totalitären Staatsform in der Sowjetunion im Besonderen konnten sich in ihrer Abwehrhaltung durch Aussagen wie beispielsweise von Wjatscheslaw M. Molotow in seiner Funktion als Volkskommissar für Auswärtige Angelegenheiten bestätigt fühlen, der auf einer Sitzung des Obersten Rates der UdSSR am 31. Oktober 1939 sagte:

Die englische Regierung hat angekündigt, daß ihr Ziel in diesem Krieg mit Deutschland nicht mehr und nicht weniger ist als die »Zerstörung des Hitlerismus«. Dies bedeutet, daß die Engländer und Franzosen so etwas wie einen »ideologischen Krieg«, der an die religiösen Kriege früherer Zeiten erinnert, an Deutschland erklärt haben [...]. Ein derartiger Krieg ist in keiner Weise gerechtfertigt. Man mag die Ideologie des Hitlerismus annehmen oder ablehnen wie jedes andere ideologische System, das ist eine Sache der politischen Anschauung. Aber jedermann sollte begreifen, daß eine Ideologie nicht durch Cewalt zerstört werden kann, daß sie nicht durch einen Krieg ausgemerzt werden kann. Es ist deshalb nicht nur sinnlos, sondern verbrecherisch, einen solchen Krieg unter der Tarnung eines Kampfes für die »Demokratie« um der »Zerstörung des Hitlerismus« willen zu führen [...] ${ }^{7}$

\title{
1.2 Frühe Renegatenberichte und ihre Erkenntnisse über das totalitäre Herrschaftssystem für die Vereinigten Staaten
}

Zweifelsohne hatte das politische Zusammengehen der »beiden großen ideokratischen Diktaturen « (François Furet) in den Vereinigten Staaten den Blick auf die Sowjetunion geschärft und den Terminus totalitär zum entscheidenden Durchbruch verholfen, um auch und vor allem den Herrschaftscharakter des stalinistischen Systems angemessen zu kategorisieren. Im Hinblick auf die sowjetische Außenpolitik in toto konnte, wie es der französische Soziologe Jules Monnerot in der bedeutenden Untersuchung Soziologie des Kommunismus konstatierte, von einer »totalitären Diplomatie« gesprochen werden. ${ }^{8}$ Die »totalitäre Diplomatie« war vor allem dadurch charakterisiert, dass sie sich nicht mehr an Abmachungen, an Verträge hielt - dies entsprach einem Wesenszug der Außenpolitik von demokratischen Staaten - und nicht bestrebt war, einen modus vivendi mit anderen Staaten zu suchen, sondern in allererster Linie eine ZweckMittel-Politik anvisierte und insofern im Kern eine unberechenbare Außenpolitik betrieb. Im Hinblick auf die stalinsche Diplomatie der späten 1930er- Jahre folgte daraus,

7 Zit. n. Arthur Koestler, Der Yogi und der Kommissar. Auseinandersetzungen, Eßlingen a. N. 1950, S. 262 f. (engl. Ausg.: The Yogi and the Commissar, London 1945).

8 Jules Monnerot, Soziologie des Kommunismus, Köln/Berlin 1952, S. 358. Der Autor nahm, wiewohl er primär den Kommunismus grundsätzlich erforschen wollte, sehr häufig eine totalitarismustheoretische Perspektive ein, weil er auch den Nationalsozialismus fokussierte. Demzufolge sprach Monnerot auf S. 367 auch von einer »totalitären Diplomatie« in Bezug auf die Außenpolitik des Nationalsozialismus. Die französische Originalausgabe erschien unter dem Titel Sociologie du Communisme im Jahre 1949. 
dass er der außenpolitischen Maxime folgte: Alles, was dem sowjetischen Staat und der KPdSU dient, kann strategisch durchgespielt und letzten Endes auch politisch realisiert werden. Es kann mit jedem Staat, ob demokratisch, autoritär oder totalitär verfasst, politisch zusammengearbeitet werden, solange es mit der sowjetischen Staatsräson kompatibel ist.

Auffallenderweise benutzen nicht wenige Autoren in ihren zeitgenössischen politischen und wissenschaftlichen Veröffentlichungen den Begriff »totalitär«. Wie gezeigt, war der Hitler-Stalin-Pakt der konkrete Anlass, sich im November 1939 auf der einflussreichen Wissenschaftskonferenz in Philadelphia aus genuin totalitarismustheoretischer Perspektive auch mit der sowjetischen Diktatur auseinanderzusetzen. ${ }^{9}$ Die aus US-amerikanischer Wahrnehmung heraus durch das deutsch-sowjetische Bündnis sich anbahnende bipolare Konfrontation zwischen amerikanischer liberaler Demokratie und den totalitären diktatorischen Staaten brachte dann der einflussreiche Totalitarismustheoretiker Franz Borkenau in seiner Untersuchung The Totalitarian Enemy 1940 prägnant zum Ausdruck, indem er feststellte, dass von den totalitären Mächten Deutschland und Sowjetunion für die westlichen Demokratien ein und dieselbe Gefahr ausgehen würde. Denn Hitler und Stalin, so Borkenau, verfolgten mit ihrer Absicht nach der Weltrevolution die gleichen imperialen außenpolitischen Ziele. ${ }^{10}$

In diesem für den weltpolitischen Verlauf des 20. Jahrhunderts ganz entscheidenden Jahr 1939 erschien die erweiterte Neuauflage der erstmalig 1935 in Paris veröffentlichten »große[n] Stalin-Biographie« (Arendt) von Boris Souvarine, die zu einem Schlüsselwerk analytisch-historisch ausgerichteter Untersuchungen zum kommunistischen Herrschaftsregime sowie totalitarismustheoretischer Arbeiten avancieren sollte. ${ }^{11}$ Souvarine kann als Prototyp des Renegaten bezeichnet werden. Eingedenk der aus heutiger Perspektive formulierten Worte aus dem philosophisch-historischen Essay Das Ende der Illusion. Der Kommunismus im 20. Jahrhundert von François Furet, dass die wirkliche Geschichte des Sowjetkommunismus »im wesentlichen von Ex-Kommunisten geschrieben werden sollte $^{12}$ stellte sich der 1895 in Kiew geborene Souvarine - 1906 nach Frankreich emigriert und Mitbegründer der Kommunistischen Partei Frankreichs (KPF) - der theoretischen Auseinandersetzung mit dem Kommunismus leninscher sowie stalinscher Couleur. Das ehemalige Mitglied des Sekretariats und des Exekutivkomitees der Kommunistischen Internationale (Komintern) wurde 1925 aus der Partei ausgeschlossen und präsentierte nach mehrjähriger intensiver Forschungsarbeit die erste politische Biografie von Jossif Wissarionowitsch Stalin, die wissenschaftlichen Standards genügte. Nicht zuletzt aus diesem Grund wurde diese Untersuchung zur »Bibel des diplomatischen Korps« [der Vereinigten Staaten] in Moskau« (Souvarine), da die veröffentlichte Stalinbiografie von Leo Trotzki einer politischen Motivation geschuldet war und somit insbesondere einen persönlich-ideo-

9 Siehe die entsprechenden Ausführungen im Einleitungskapitel.

10 Borkenau, The Totalitarian Enemy. Vgl. die als wissenschaftliche Vorarbeit zu dieser Studie einzuschätzende Untersuchung von Borkenau, The Communist International, London 1938. In den Vereinigten Staaten erschien das Buch unter dem Titel World Communism. A History of the Communist International in New York.

11 Boris Souvarine, Stalin. A Critical Survey of Bolshevism, New York 1939 (dt. Ausg.: Stalin. Anmerkungen zur Geschichte des Bolschewismus, Amsterdam 1940).

12 Furet, Das Ende der Illusion, S. 191. 
logischen Abrechnungscharakter besaß. ${ }^{13}$ Mithilfe der Untersuchung von Souvarine über den kommunistischen »Großinquisitor sui generis« bestand die Möglichkeit, die Entstehungsgeschichte und die Verlaufsform der Russischen Revolution von 1917 nachzuzeichnen. Es war der Versuch, anhand marxistischer Kategorien eine Erklärung zu finden für die gescheiterte Oktoberrevolution und vor allem das bis zu diesem Zeitpunkt »ungelöste Geheimnis«, das »ungeklärte Rätsel« um den absoluten Diktator und allmächtigen Führer der KPdSU zu lüften. Zudem besaß die bis dahin »umfassendste Biographie Stalins« (Walter Krivitsky) von Souvarine insofern noch eine zentrale Bedeutung, als hiermit auf der Grundlage einer ersten Schätzung - grosso modo - zuverlässige Opferzahlen der stalinschen Industrialisierungs- und Kollektivierungspolitik mitgeteilt wurden. Nach einer »hervorragenden vertraulichen Quelle der GPU«, so Souvarine, ergab eine sowjetische Volkszählung aus dem Jahre 1937, dass Stalins Politik annähernd 30 Millionen Menschenleben gekostet haben dürfte. ${ }^{14}$

Die sogenannten Russlandexperten im State Departement der Vereinigten Staaten, d.h. in erster Linie die "Riga-Fraktion« - von der weiter unten die Rede sein wird - konnten sich in ihrer frühzeitig konstituierenden skeptischen Haltung gegenüber dem »totalitären Kommunismus« (Souvarine) bestätigt fühlen. Denn in ihren Augen existierte ein Konnex zwischen der Russischen Revolution von 1917, dem terroristischen Verlauf des erfolgreichen Umsturzes in Moskau mit den begangenen Verbrechen und der russischen Tradition. Für die Russlandkenner und -forscher um den Diplomaten und Historiker George F. Kennan waren die Massenverbrechen in der Folge der kommunistischen Revolution keineswegs ein Kontingenzphänomen, sondern entsprangen systemimmanenten Ursachen. Gleichwohl existierte keine historische Notwendigkeit für den prätotalitären Lauf der kommunistischen Dinge in Russland. Diese Einschätzung teilten der Renegat Souvarine und die insbesondere im Hinblick auf die amerikanische Außenpolitik nach Beendigung des Zweiten Weltkrieges einflussreiche »Riga-Fraktion«.

Kurze Zeit nach der Unterzeichnung des weltweit als absolute Überraschung begriffenen Hitler-Stalin-Paktes erschienen unter anderem durch die Initiative von Isaac Don Levine - einem gebürtigen Russen und prominenten New Yorker antistalinistischen Verleger - die Erinnerungen In Stalins Secret Service vom ehemaligen Chef des sowjetischen Nachrichtendienstes in Westeuropa und einem der wichtigsten Mitarbeiter des Untergrundapparates (bzw. des Geheimdienstes im Ausland) Walter G. Krivitsky. Der Band, der zeitgleich in London unter dem Titel I was Stalin's Agent ${ }^{15}$

13 Der spätere Biograf Stalins, Paloczi-Horvath, attestierte Souvarine »der Verfasser der ersten ausgezeichneten politischen Biographie von Stalin« gewesen zu sein, der eine »realistische[...] Stalin-Charakterisierung gegeben hat« (Ceorge Paloczi-Horvath, Stalin, Zürich 1968, S. 103).

14 Boris Souvarine, Stalin. Anmerkungen zur Geschichte des Bolschewismus, München 1980, S. 599 (hierbei handelt es sich um die deutsche Neuausgabe). Der Autor bezog sich auf Kriwitzkis Informationsmaterial, ohne eine genaue Quellenangabe zu machen. Vgl. hierzu auch Arendt, Elemente und Ursprünge totaler Herrschaft, S. 501, Anm. 13.

15 Walter G. Krivitsky (d. i. Samuel Ginsberg), In Stalin's Secret Service. Memoirs of the first soviet master spy to defect, New York 1939 (engl. Ausg.: In Stalins Secret Service. An Expose of Russias Secret Service Policies by the Former Chief of the Soviet Intelligence of Western Europe, London 1939; dt. Ausg.: Ich war in Stalins Dienst!, Amsterdam 1940). In Frankreich erschien der Band unter dem Titel Agent de Staline in Paris 1940. Im selben Jahr wurde der Band in einer holländischen Übersetzung vorgelegt sowie in einer italienischen Fassung vom faschistischen System unter Mussolini. 
verlegt wurde, zählte einerseits zu einem der ersten autobiografischen Renegatenberichte - Krivitsky vollzog den Bruch mit der Kommunistischen Partei stalinscher Provenienz nach einem mehrjährigen Prozess des Zweifelns endgültig im Herbst 1937 und besaß andererseits bereits zum damaligen Zeitpunkt eine herausragende Bedeutung, insofern der Verfasser als führender Offizier und Aktivist des sowjetischen Geheimdienstapparates mit dem facettenreichen stalinistischen Terror der 1930er-Jahre bestens vertraut war und infolgedessen eine erste annähernde Beschreibung über die für Außenstehende schwer zu durchschauende Rolle und die Methoden des NKWDs inner- und außerhalb der Sowjetunion liefern konnte. ${ }^{16}$

Der unter dem Namen Samuel Ginsberg 1899 in der Ukraine geborene spätere Renegat schloss sich bereits mit 13 Jahren der russischen Arbeiterbewegung an und trat in die Kommunistische Partei nach dem Ersten Weltkrieg im Jahre 1919 ein. Nach überaus erfolgreicher Aktivität in der Roten Armee während des russischen Bürgerkrieges durchlief Ginsberg im Auftrage der Armeeführung verschiedene Ausbildungskurse, in denen er Erfahrung mit dem Einmaleins des militärischen Nachrichtendienstes machte. 1923 wurde er unter dem Tarnnamen Walter Krivitsky zur Vorbereitung der kommunistischen Revolution in den Untergrund nach Deutschland geschickt und war als einer der ranghöchsten Offiziere des militärischen Geheimdienstes maßgeblich für die Sowjetisierung der Kommunistischen Partei Deutschlands (KPD) verantwortlich. Stalin, der bereits auf seinem Weg zur unumschränkten Macht durch seine erbarmungslose Kollektivierungspolitik zu Beginn der 1930er-Jahre Millionen Menschenleben durch das vorsätzliche Zurückhalten staatlicher Hilfe, so Krivitsky, in Kauf nahm, ${ }^{17}$ avancierte nicht zuletzt aufgrund seiner moralisch und außenpolitisch verhängnisvollen Rolle im Spanischen Bürgerkrieg und im Kontext der Moskauer Schauprozesse zum Phänotyp eines »totalitären Despoten«, sodass die spätere Zusammenarbeit mit dem Nationalsozialismus keineswegs dem Zufall, der Naivität oder dem mangelnden Urteilsvermögen der kommunistischen »Nummer eins « geschuldet war. ${ }^{18}$

In einem zentralen Abschnitt seines autobiografischen Renegatenbuches ging Krivitsky einer sich durch die drei öffentlichen Moskauer Schauprozesse zwangsläufig aufdrängenden Frage nach. Diese Frage stellte sich vielen Beobachtern und interessierten Zeitgenossen der Prozesse, die die Natur des sowjetischen Totalitarismus fokussierten: „Warum hatten sich die alten Bolschewiken, Helden und Führer der Revolution, die mit dem Tode so oft in Berührung gekommen waren, daß sie sich selbst >Tote auf Urlaub< nannten, zu diesen absurden und haarsträubenden

16 Des Weiteren besaß das Kriwitzki-Buch eine zusätzliche herausragende Bedeutung, als hiermit zum ersten Male ein exponierter Funktionär des russischen Geheimdienstes mit der stalinschen Politik und der Kommunistischen Partei unter Stalin brach und einen persönlichen Erfahrungsbericht veröffentlichte. Erst mehrere Jahre später erschien der Band des ehemaligen führenden NKWD-Offiziers Alexander Orlow, The Secret History of Stalin's Crimes, New York 1953 (dt. Ausg.: Kremlgeheimnisse, Würzburg 1953). Orlow, 1938 in die USA geflüchtet, schilderte hier detaillierte Einzelheiten zur stalinschen Politik; insbesondere beleuchtete er anhand seiner persönlichen Erkenntnisse die »Croßen Säuberungen « und die Moskauer Schauprozesse in den 1930er-Jahren.

17 Krivitsky, Ich war in Stalins Dienst!, S. 9.

18 Auf diesen für die autobiografischen Erfahrungsberichte vieler Exkommunisten zentralen Gesichtspunkt der zeitlichen Koinzidenz des Spanischen Bürgerkrieges - mit dem expliziten stalinistischen Terror in den Reihen der Republikanischen Front - und den Moskauer Schauprozessen sowie der politischen Bedeutung ebendieser historischen Phänomene komme ich in Kap. II.2 zurück. 
Lügen bekannt? «19 Für den Bolschewisten der ersten Stunde und frühen Exponenten des kommunistischen Apparates Krivitsky waren die Geständnisse in persona des fast gleich alten Sergej Mratschkowskij - einem von insgesamt 16 Angeklagten im ersten Schauprozess vom 19. bis 24. August 1936 -, der beschuldigt wurde, Mitglied eines sogenannten antisowjetischen vereinigten trotzkistisch-sinowjewschen Zentrums zu sein, keinesfalls das weithin unerklärliche große Rätsel. Durch Krivitsky erfuhr die amerikanische Öffentlichkeit, was sich hinter den »Kulissen dieses Puppentheaters« des Generalstaatsanwaltes Andrei Wyschinski cum grano salis tatsächlich abspielte. Neben den physischen und psychischen Foltermethoden der russischen Geheimpolizei stand für Krivitsky fest - der mit Mratschkowskij und den anderen Angeklagten die ideologischen Grundüberzeugungen teilte und aufgrund einer politischen Mentalitätsverwandtschaft imstande war, die psychischen Prozesse der Altbolschewiken zu verstehen und mithin zu interpretieren -:

Obwohl es mancherlei Faktoren waren, welche diese Männer zu solchen Ceständnissen veranlassten, so haben sie doch alle zweifellos in der aufrichtigen Überzeugung gehandelt, dass sie der Partei und der Revolution diesen letzten Dienst leisten müssten. Sie opferten Ehre und Leben zur Verteidigung des verhassten Stalin-Regimes, weil sie in ihm den letzten schwachen Hoffnungsschimmer für jene bessere Welt sahen, der sie sich in ihrer frühen Jugend geweiht hatten. ${ }^{20}$

Krivitsky wandte sich frühzeitig im Laufe des Jahres 1939 an die US-amerikanische Öffentlichkeit in Form mehrerer Zeitungsartikel in der New Yorker Saturday Evening Post, um über den Stalinismus im Allgemeinen und die sowjetische Außenpolitik im Besonderen $\mathrm{zu}$ informieren. Zudem intendierte er, vor allem auf die Gefahren hinzuweisen, die der bevorstehende Hitler-Stalin-Pakt evoziere. Die Artikel, die von seinem Mitarbeiter Boris Shub ${ }^{21}$ ins Amerikanische übersetzt wurden, sollten die Vereinigten Staaten warnen, denn Krivitsky beurteilte die Aussichten mehrere Monate vor der endgültigen offiziellen deutsch-sowjetischen-Zusammenarbeit im Gegensatz zu den allermeisten westlichen Politikern und Zeitgenossen als überaus realistisch. ${ }^{22}$

19 Arthur Koestler, Sonnenfinsternis. Roman. Aus dem Engl., Frankfurt a. M./Berlin/Wien 1979, S. 228.

20 Krivitsky, Ich war in Stalins Dienst!, S. 210.

21 Boris Shub, Sohn von David Shub (Freund und Cesprächspartner von Kriwitzki), sollte ein bekannter Sowjetforscher werden und u. a. regelmäßig Artikel für die Zeitschrift Der Monat schreiben. David Shub wurde in Russland geboren, war seit 1903 Mitglied der russischen Sozialdemokratischen Partei und zum Zeitpunkt von Lenins Führung Aktivist der Revolution von 1905. Er wurde verhaftet und nach Sibirien in die Verbannung geschickt. Ihm gelang die Flucht in die Vereinigten Staaten und er hatte ständigen Kontakt mit menschewistischen und bolschewistischen Politikern wie zum Beispiel Kerenski bzw. Lenin, Trotzki und Bucharin. David Shub veröffentlichte im Jahre 1948 in New York eine vielgelesene und -beachtete Biografie über Lenin; Teilabdruck in: Der Monat 1 (1949), H. 3, S. 37-48.

22 Krivitsky teilte mit, dass seit Januar 1937 Stalin über den verantwortlichen (offiziellen) sowjetischen Handelsvertreter in Berlin, David Kandelaki, Hitler das Angebot einer Zusammenarbeit machte, an dem dieser sich zu diesem für ihn frühen Zeitpunkt indes noch uninteressiert zeigte. Der damalige Geheimdienstchef Nikolai Jeschow sagte zu Kriwitski: »Deutschland ist stark. Die Deutschen sind die stärkste Macht der Welt. Hitler hat sie dazu gemacht. Wer könnte daran zweifeln? Muß nicht jeder, der bei Verstand ist, das in seine Pläne einbeziehen? Es gibt nur eine Strategie für die Sowjetunion.« Sodann behauptete Jeschow, laut Kriwitski, dass Stalin davon sprach, dass »wir uns mit einer Super- 
Gleichwohl wurde nicht nur die amerikanische Öffentlichkeit durch den aus der Sowjetunion geflüchteten Krivitsky ${ }^{23}$ informiert, sondern auch das US State Department und das FBI. Denn die Vereinigten Staaten wurden nach seinem Pariser Aufenthalt zu seinem Flucht- und Exilland. Die amerikanischen staatlichen Dienststellen wurden en detail nicht zuletzt über die Infiltrationsaktivitäten der Sowjetunion informiert, die es geschafft hatten, NKWD-Agenten an wichtigen Stellen der US-Administration zu platzieren.

Anlässlich der Buchveröffentlichung bzw. der Artikelserie in der Saturday Evening Post im Jahre 1939 und der damit verbunden unerwarteten starken Rezeption insbesondere bei der antistalinistischen Linken in New York konkretisierte Krivitsky die Beschreibung und Analyse des stalinistischen Totalitarismus - wiewohl der Kampf gegen jedweden politischen Totalitarismus ging - auf verschiedenen Pressekonferenzen in den Vereinigten Staaten. Infolgedessen wurde ein Ablösungsprozess vieler Intellektueller und Sympathisanten von der Kommunistischen Partei der Vereinigten Staaten (KPUSA) vor allem durch die genaue Beschreibung der verhängnisvollen stalinschen Außenpolitik im Spanischen Bürgerkrieg in Gang gebracht. Gleichzeitig starteten die Kommunisten in der amerikanischen Öffentlichkeit eine immense Verleumdungskampagne gegen Krivitsky. Nachdem der NKWD-Apparat bereits mehrere Abtrünnige der kommunistischen Bewegung im Ausland umgebracht hatte und zwei Versuche, Krivitsky zum Zeitpunkt seines Aufenthalts in Frankreich zu ermorden, fehlschlugen, wusste der ehemalige Geheimdienstfunktionär, dass er auch in den Vereinigten Staaten konkret von Stalins Terrormaschinerie bedroht war. Am 11. Februar 1941 informierte die New York Times darüber, dass ein Tag zuvor Krivitsky unter mysteriösen Umständen mit einem Kopfschuss tot in einem Washingtoner Hotelzimmer aufgefunden wurde. ${ }^{24}$

Im Jahre 1940 erschienen in London zwei Bücher von zwei ehemaligen Anhängern der kommunistischen Bewegung und der kommunistischen Idee, die auch in den Vereinigten Staaten von nicht geringer Bedeutung waren. Beide Autoren zählten vor ihrem Bruch zu führenden (Partei-)Intellektuellen respektive Funktionären der Kommunistischen Partei. Sie präsentierten unabhängig voneinander sozusagen autobiografische Erfahrungsberichte aus erster Hand, die zweifelsohne die politisch-wissenschaftliche und intellektuelle Auseinandersetzung über die Sowjetunion und das stalinistische Herrschaftsregime auf eine neue Stufe stellten. Die Rede ist zum einen

macht wie Nazi-Deutschland einigen müssen«. Zit. n. Christopher Andrew, Oleg Gordiewsky, KGB. Die Geschichte seiner Auslandsoperationen von Lenin bis Corbatschow, München 1990, S. 299.

23 Krivitsky beantragte unter Todesdrohung in Paris am 7. Dezember 1937 politisches Asyl und musste miterlebten, wie sein Genosse und Freund, der ehemalige GPU-Agent und Renegat Ignaz Reiss, kurz zuvor durch den sowjetischen Ceheimdienst in der Schweiz umgebracht wurde.

24 Siehe zu den Umständen des Todes, der von der amerikanischen Polizei nicht zuletzt aufgrund von drei gefundenen Abschiedsbriefen von Krivitsky als Selbstmord apostrophiert wurde, im Einzelnen: Hellmut C. Haasis (Hg.), Walter C. Krivitsky: Ich war Stalins Agent, Grafenau-Döffingen 1990, S. 297 ff. Krivitsky erklärte indes mehrmals gegenüber seiner Familie und Freunden, dass, sollte er eines fernen Tages Tod aufgefunden werden, ein Selbstmord ausgeschlossen werden kann. 
von dem Buch The Russian Enigma von Ante Ciliga ${ }^{25}$ und zum anderen von Arthur Koestlers Darkness at Noon ${ }^{26}$.

Der 1898 in Istrien geborene Anté Ciliga trat 1918 als Student in die Sozialistische Partei Kroatiens ein, nachdem er zuvor im Ersten Weltkrieg noch aufseiten der kroatischen nationalistischen Bewegung mitkämpfte. Er gehörte zu den Mitbegründern der Jugoslawischen Kommunistischen Partei (KPJ) und avancierte dort 1922 zum Parteisekretär für Kroatien. Nachdem Ciliga in der Balkanabteilung der Komintern in Wien arbeitete, wurde er im Oktober 1926 in die Sowjetunion beordert. Aufgrund der konkreten Erfahrungen mit der kommunistischen Wirklichkeit infolge eines mehrjährigen Aufenthaltes in Moskau und Leningrad begann daraufhin sein Ablösungsprozess vom Kommunismus. Allmählich stellte er das stalinistische Herrschaftsregime infrage. 1930 wurde er aufgrund seiner Kritik an den sowjetischen Zuständen verhaftet und für drei Jahre in das Gefängnis Werchneuralsk und danach in ein sibirisches Internierungslager gesteckt. Im Dezember 1935 ermöglichte Ciliga die Berufung auf eine italienische Staatsbürgerschaft die Sowjetunion zu verlassen, um danach in Frankreich zu leben; in Paris erschien sodann 1938 sein Erinnerungsbuch Au pays du grand mensonge. ${ }^{27}$ Die Untersuchung sollte in mehrfacher Hinsicht Bedeutung erlangen, denn neben einer gründlichen Darstellung und Interpretation der Moskauer Schauprozesse versuchte der Autor, sich dem Phänomen des Massenterrors in der Sowjetunion der 1930er-Jahre zu stellen und analytisch anzunähern, der sich keineswegs aus dem gesellschaftlichen und politischen Nichts heraus entwickelte. Insbesondere beleuchtete Ciliga den Konnex zwischen Stalins Herrschaftstechnik und -politik sowie der impliziten Rolle der Geheimpolizei. Er hob hervor, dass Stalin den Geheimdienstapparat bereits im Verlauf des Machtkampfes um die Nachfolge Lenins zu einem zentralen Herrschaftsinstrument seiner Politik machte und mithin einen entscheidenden Schritt auf dem Weg zur genuinen totalitären Bewegung zurücklegte. Zentrale Bedeutung erlangte die Untersuchung Ciligas in Bezug auf die Sowjetforschung sowie auf totalitarismustheoretische Arbeiten wie beispielsweise Arendts Elemente und Ursprünge totaler Herrschaft vor allem deshalb, weil er einerseits die fundamentale Tragweite des Arbeitslagersystems für den sowjetischen Staat ernsthaft in den (Forschungs-)Blick nahm und weil er auf den bedeutenden Umstand hinwies, dass der Terror in der Sowjetunion nicht nach der Verfolgung von Sozialisten, Anarchisten, der Bauernschaft oder sonstigen vermeintlichen oppositionellen Personen und Gruppen ein Ende fand. Ganz im Gegenteil: Der entscheidende Unterschied des sowjetischen Herrschaftssystems unter Stalin im Vergleich zu traditionellen Herrschaftsregimen wie zum Beispiel der Tyrannei bestand eben gerade darin, dass der systematische stalinistische Massenterror nämlich erst zu dem Zeitpunkt sich radikal Bahn brach, als

25 Anté Ciliga, The Russian Enigma, London 1940. Der Band erschien erstmals unter dem Titel Au pays du grand mensonge in Paris 1938 (dt. Ausg.: Im Land der verwirrenden Lüge. Zehn Jahre hinter dem Eisernen Vorhang, Köln 1953).

26 Arthur Koestler, Darkness at Noon, London 1940 (dt. Ausgabe: Sonnenfinsternis, Stuttgart 1948). Im zweiten Band seiner Autobiografie schrieb Koestler im Hinblick auf die Bedeutung der Erfahrungsberichte von Exkommunisten, die nicht selten »Apparat-Leute« waren, dass zum Beispiel das Buch von Ciliga u. a. die Hauptquelle darstellte, »der der Westen die Kenntnis seines Cegners verdankt« (Arthur Koestler, Abschaum der Erde. Autobiographische Schriften. Bd. 2, Berlin/München 1993, S. 257).

27 Furet, Das Ende der Illusion, S. 675, Anm. 38. Ebd. auch nähere Angaben über die Biografie Anté Ciligas. 
die reale respektive vermeintliche Gegnerschaft bereits besiegt war. Das heißt, "nicht in den zwanziger Jahren und zu Zeiten des Bürgerkriegs in Rußland, sondern nach 1930, als alle gegenrevolutionäre wie innerparteiliche Opposition restlos liquidiert war, brach die wirkliche Schreckensherrschaft in Rußland an ${ }^{28}$

$\mathrm{Zu}$ einem der zweifelsohne weltweit einflussreichsten kommunistischen Renegaten der 1940er- und 1950er-Jahre des 20. Jahrhunderts zählte Arthur Koestler. Koestler, der im Laufe einer mehrjährigen Auseinandersetzung - die zum Teil existenzielle Dimensionen erreichte - aufgrund einer intensiven Auseinandersetzung mit theoretischen und politisch-lebenspraktischen Fragen der kommunistischen revolutionären Idee schlussendlich 1938 im Anschluss an seine persönlichen Erfahrungen im Kontext des Spanischen Bürgerkrieges mit der Kommunistischen Partei brach, veröffentlichte daraufhin sein »sensationelles « Buch Ein spanisches Testament ${ }^{29}$. Sein 1940 erschienener Roman Darkness at Noon (deutscher Titel: Sonnenfinsternis) wurde der klassische literarische Text über die berüchtigten stalinistischen Schauprozesse, in dem der Autor versuchte, das Rätsel der Selbstanklage anhand der Bucharin nachgezeichneten Figur des Rubaschow zu beschreiben und zu entziffern. ${ }^{30}$ Das Buch begründete Koestlers Rang des Prototypus eines antikommunistischen und - aufgrund einer ansatzweise totalitarismustheoretischen Perspektive - antitotalitären Renegaten. Bemerkenswerterweise arbeitete Koestler mittels des Romans unter anderem die Verkoppelung von Ideologie und Terror heraus, um das Phänomen der permanenten Gewalt und Säuberung zu erklären.

Im Verlauf der nächsten Jahre bis zum Ende des Zweiten Weltkrieges wandte sich Koestler in mehreren Zeitschriftenartikeln sowohl an die englische als auch an die amerikanische interessierte Öffentlichkeit. Neben aktuellen tagespolitischen Themen beinhalten die in Form von Essays publizierten Arbeiten neben politisch-analytischen Reflexionen über die vom nationalsozialistischen Gewaltregime begangenen Massenverbrechen an den europäischen Juden insbesondere die Thematisierung und die Untersuchung des sowjetkommunistischen Herrschaftssystems. Den Schwerpunkt setzte Koestler in der Analyse der überragenden Rolle Stalins in Bezug auf die Innenund Außenpolitik der 1930er- und 1940er-Jahre, der Funktion der Geheimpolizei, der Bedeutung der Zwangsarbeit und des Arbeitslagersystems für das ökonomische und politische System in der Sowjetunion. Zudem rückten auch und vor allem der stalinistische Massenterror, die Parteisäuberungen und die Schauprozesse in den Fokus des (Forschungs-)Interesses von Koestler. Ein weiterer Schwerpunkt war die Komplizen-

28 Arendt, Elemente und Ursprünge totaler Herrschaft, S. 616, die sich hier ausdrücklich auf das Buch von Anté Ciliga bezog. Überhaupt bezog sich Arendt in der zum Schlüsselwerk der Totalitarismusforschung gewordenen Untersuchung in dem dritten Teil an vielen Stellen auf das Werk von Ciliga. Zudem ist die Studie zweifelsohne ohne die zahlreichen Renegatenberichte, auf die Arendt insbesondere in Bezug auf die Analyse des Sowjetkommunismus rekurrierte, nicht vorstellbar.

29 Arthur Koestler, Ein spanisches Testament. Aufzeichnungen aus dem Bürgerkrieg, Zürich 1938.

30 Auf die überaus interessante zeitliche und ideologiekritische Koinzidenz mit Kriwitzkis Buch I was Stalin's Agent und dessen Versuch, die Ceständnisse der Angeklagten im Rahmen der Moskauer Schauprozesse zu erhellen, machte Koestler in den persönlichen Anmerkungen in der deutschen Ausgabe seines Romans deutlich. Er konstatierte, dass die »Ähnlichkeit der Atmosphäre und des Inhalts« in den beiden Büchern auffielen, obwohl Krivitsky Sonnenfinsternis nicht gelesen habe und Koestler den autobiografischen Renegatenbericht von Krivitsky erst mehrere Jahre nach seiner eigenen Veröffentlichung rezipierte (Koestler, Sonnenfinsternis, S. 235 f.). 
schaft zwischen den beiden totalitären Herrschaftsregimen des deutschen Nationalsozialismus und des Sowjetkommunismus unter Stalin sowie der implizite und explizite Systemvergleich der beiden diktatorischen Regime. ${ }^{31}$

Dass die sowjetische Politik bereits zu Beginn der 1940er-Jahre für die amerikanische Öffentlichkeit und die staatlichen Regierungsstellen alles andere als eine terra incognita blieb, dafür sorgte - neben den Publikationen wie beispielsweise von Koestler - in allererster Linie ein weiterer Insider aus dem kommunistischen Machtapparat. Im Januar 1941 veröffentlichte der 1905 in Darmstadt geborene Richard Krebs unter dem Autorennamen Jan Valtin in New York seinen autobiografischen Renegatenbericht Out of the Night ${ }^{32}$. Das Buch sorgte in den Vereinigten Staaten für gewaltiges Aufsehen. Die Auflagenhöhe betrug bereits bis zum März 1941 knapp 347000 und bis 1951 insgesamt über eine Million Exemplare. Neben mehreren Veröffentlichungen von Krebs in renommierten amerikanischen Zeitschriften wurde die aufsehenerregende Bucherscheinung beispielsweise von Interviews und Rundfunksendungen mit Krebs sowie Teilabdrucken in englischen und amerikanischen Periodika begleitet. Ende 1941 sollte Out of the Night nach Einschätzung der Times neben Koestlers Darkness at Noon zu den Büchern des Jahres gehören.

Die zentrale Bedeutung des Bestsellers lag insbesondere für die amerikanischen Regierungsstellen darin, dass infolge der krebschen Mitteilungen ein weiteres Mal genaue Einzelheiten über das stalinistische Herrschaftssystem ans Tageslicht kamen; hinzu kamen die selbstverständlich ebenfalls auf Interesse gestoßenen reflexiven und beschreibenden Passagen über den Herrschaftscharakter und die -praxis des Nationalsozialismus in den 1939er-Jahren. Krebs war ein ehemaliger Funktionär aus dem tiefen »Dschungel« des kommunistischen Untergrundapparats in Deutschland sowie der Komintern und deshalb geradezu prädestiniert, Erklärungen zu tätigen über die totalitäre Politik der Sowjetunion, den Zusammenhang zwischen der Kominternpolitik und der Politik der KPD in den 1920er- und 1930er-Jahren sowie en détail über die überaus wirkungsvolle menschenvernichtende Terrorpraxis der stalinistischen Geheimpolizei. Zudem konnte der Renegat Krebs die offiziellen staatlichen Stellen der Vereinigten Staaten über die sehr realen Gefahren informieren, die angesichts der kommunistischen Agenten entstanden waren. Das heißt, es ging hier nicht zuletzt auch um die ernst zu nehmenden Risiken einer überaus effektiven »fünften Kolonne« in den USA. Auf allerhöchster staatlicher Ebene benutzte man die Detailkenntnisse von Krebs alias Jan Valtin, um dem intakten Agentennetz auf die Spur zu kommen. Als Zeuge sagte er im Mai 1941 vor dem gemeinhin als »Dies-Committee« apostrophierten House Un-American Activities Committee (HUAC) aus. ${ }^{33}$ Eingedenk des Hitler-

31 Koestler, The Yogi and the Commissar, London 1945.

32 Jan Valtin (d. i. Richard Krebs), Out of the Night, New York 1941 (dt. Ausg.: Tagebuch der Hölle, Köln 1957). Vgl. zu Richard Krebs insbesondere das Kapitel »Richard Krebs: Der Kominternagent«in: Michael Rohrwasser, Der Stalinismus und die Renegaten. Die Literatur der Exkommunisten, Stuttgart 1991, S. 177-231. Im Folgenden beziehe ich mich auf diese Renegatenstudie. Vgl. zu Krebs die Biografie von Ernst von Waldenfels, Der Spion, der aus Deutschland kam. Das geheime Leben des Seemanns Richard Krebs, Berlin 2002 sowie Hermann Kuhn, Bruch mit dem Kommunismus. Über autobiographische Schriften von Ex-Kommunisten im geteilten Deutschland, Münster 1990, S. 21 f.

33 Das HUAC wurde vom Repräsentantenhaus konstituiert und begann im August 1938 mit seinen Aktivitäten. Als Vorsitzender fungierte der Südstaatler und Rechtsaußen der Demokratischen Partei Martin Dies. Es stellte die historisch-politische Vorwegnahme des nach dem Zweiten Weltkrieg ins 
Stalin-Paktes konnte Out of the Night zu Beginn des Jahres 1941 auch deshalb in den Vereinigten Staaten für (politische) Furore sorgen, weil der Exkommunist Krebs einen impliziten totalitarismustheoretischen Blick einnahm. Das will heißen: Neben den mörderischen Aktivitäten der »deutschen Tscheka« bezog Krebs - der zeitweise nach seiner Verhaftung durch die Gestapo im Jahre 1933 im deutschen Gefängnis saß - das nationalsozialistische Herrschaftssystem im Allgemeinen und die Gestapo im Besonderen in seine zum teil analytischen Schilderungen mit ein. Hierzu schreibt Michael Rohrwasser angesichts der politischen Zusammenarbeit zwischen der NSDAP und der KPD während der Weimarer Republik:

Nicht allein darum mißt Krebs der KPD eine Schuld bei am Zustandekommen der sogenannten Machtergreifung Hitlers. Er erklärt die Machenschaften des kommunistischen Untergrundapparates zu einem Spiegelbild der Cestapo. Er illustriert das, was Bertolt Brecht 1934 im Cespräch mit Walter Benjamin sagte: »Was aus der Tscheka werden kann, sieht man an der Gestapo«. Indem Krebs den Komintern-Apparat, die GPU und die anderen kommunistischen Apparate als Vorläufer des nazistischen Apparats versteht, zerstört er das große Entweder-Oder, den stalinistischen Mythos des alles legitimierenden Antifaschismus. Er unterstreicht mit Beispielen [...] die mit tödlicher Effektivität funktionierende Zusammenarbeit von Gestapo, GPU und Komintern. ${ }^{34}$

Leben gerufenen berüchtigten McCarthy-Ausschuss gegen »antiamerikanische Umtriebe«dar. Krebs zählte neben der ehemaligen deutschen Kommunistin und Emigrantin Ruth Fischer, die sich später durch die Untersuchung "Stalin and German Communism«, New York 1948 (dt. Ausg.: Stalin und der deutsche Kommunismus: Der Übergang zur Konterrevolution, Frankfurt a. M. o. J.) einen Namen machte, Kriwitzki, dem kommunistischen Kandidaten für die amerikanische Vizepräsidentschaft von 1924 und 1928 sowie Autor des 1940 in New York erschienen Buches I Confess. The Truth about American Communism, Benjamin Gitlow, dem ehemaligen Kommunisten und Mitarbeiter des Frankfurter Institutes für Sozialforschung Karl August Wittfogel, zu den prominentesten Zeugen.

34 Michael Rohrwasser über Valtins Buch Tagebuch der Hölle (unveröff. Vortragsmanuskript vom Oktober 1993, S. 7). Zu der Totalitarismustheorie in nuce passt das vermutlich vom Verleger Joseph Caspar Witsch verantwortete Vorwort der deutschen Ausgabe von 1957. Er brachte hier zum Ausdruck, dass das Renegatenbuch von Krebs als ein eindrückliches essenzielles Plädoyer gegen jedweden Versuch qua die Kategorien »Klasse oder Rasse« eine»Tyrannei totalitärer Ordnung «zu etablieren, verstanden werden kann. Er stellte aufS. 5 fest: »Das vorliegende Buch ist das Zeugnis eines von totalitären Systemen vielfach gemarterten und verfolgten Menschen, ein Zeugnis auch für die erschreckende Wahrheit über diese Systeme.«Zudem warnte er ausdrücklich die westlichen Demokratien angesichts der innen- und außenpolitischen Gefahren durch die mit neuartigen Herrschaftsmethoden operierenden totalitären Regime, die sich nicht davon abhalten ließen, offenen und heimlichen Terror durch ihre geheimdienstlichen Apparate zu praktizieren, und diese menschenverachtende Gewalt zudem auch ins Ausland übertrugen. Darüber hinaus ist festzuhalten, dass Krebs als antikommunistischer und antitotalitärer Renegat, der im Frühjahr 1947 nach langem Prozedere die US-Staatsbürgerschaft erhielt, dem amerikanischen Gesellschaftssystem keineswegs kritiklos verfiel, sondern ihm ein »unpathetisches ]a« entgegenbrachte (vgl. hierzu auch Rohrwasser, Der Stalinismus und die Renegaten, S. 208). 